Full-text Available Online at www.ajol.info and www.bioline.org.br/ja
J. Appl. Sci. Environ. Manage. June., 2016

Vol. 20 (2) $350-354$

\title{
Stability Analysis of the Endemic Equilibrium State of an Infection Age-Structured HIV/AIDS Disease Pandemic
}

\author{
${ }^{1}$ ASHEZUA, TT; ${ }^{2}$ UDOO, IJM; ${ }^{3}$ IKPAKYEGH, LN \\ ${ }^{1,3}$ Department of Mathematics/Statistics/Computer Science, Federal University of \\ Agriculture, Makurdi, Nigeria \\ ${ }^{1}$ Email: ttashezua@gmail.com, ${ }^{3}$ Email: lewisnyitor1984@gmail.com, \\ ${ }^{2}$ Department of Mathematics, School of Sciences, College of Education, Zing, Nigeria \\ ${ }^{2}$ Email: iorlumunudoo@gmail.com
}

\begin{abstract}
In this work we present an infection-age-structured mathematical model of AIDS disease dynamics and examine the endemic equilibrium state for stability. An explicit formula for the basic reproduction number $R_{0}$ was obtained in terms of the demographic and epidemiological parameters of the model. The endemic equilibrium state was found to be locally asymptotically stable under certain conditions. Furthermore, by constructing a suitable Lyapunov functional, the endemic equilibrium state was found to be globally asymptotically stable under certain conditions prescribed on the model parameters. (C) JASEM
\end{abstract}

http://dx.doi.org/10.4314/jasem.v20i2.16

KEYWORDS: Basic reproduction number, HIV/AIDS, Lyapunov functional

\section{INTRODUCTION}

This paper presents an infection-agestructured mathematical model of the AIDS disease dynamics proposed by Akinwande (2005). The population considered was partitioned into the Susceptibles $S(t)$ and the infected $I(t)$. The susceptible individuals are those who are free from HIV/AIDS but are open to get the virus as they interact with those who are infected while the infected individuals are those who have contracted the virus and are at various stages of infection. The infected class was structured according to time and age of infection $\rho(t, \tau)$, where $t$ the time parameter and $\tau$ is the infection age. They assumed that while the new births in the susceptible class $S(t)$ are born therein, the off-springs of the infected $I(t)$ are divided between $S(t)$ and $I(t)$ in the proportions $\theta$ and

$\frac{d S(t)}{d t}=(\beta-\mu) S(t)+\theta \beta I(t)-\alpha S(t) I(t)$ $(1-\theta)$ respectively. This implied that a proportion $(1-\theta)$ of the off-springs of the infected are born with the virus. In Akinwande (2005) a characteristic equation was obtained and analysed using the results of Bellman and Cooke (1963).This paper presents a variant of the model by Akinwande (2005) and we study the stability analysis of the endemic equilibrium state using the method of linearization and the Lyapunov method. The model equations are presented in section two while the results and discussion in section three.

\section{MATERIAL AND METHODS}

We reproduce the model by Akinwande (2005) on 'a mathematical model perspective of chaos in the dynamics of AIDS disease pandemic as follows:

$$
\frac{\partial \rho(t, \tau)}{\partial t}+\frac{\partial \rho(t, \tau)}{\partial \tau}+(\mu+\sigma(\tau)) \rho(t, \tau)=0
$$




$$
\begin{aligned}
& \sigma(\tau)=\delta_{1} e^{-k(T-\tau)} \\
& I(t)=\int_{0}^{T} \rho(t, \tau) d \tau \\
& \rho(t, 0)=B(t)=\alpha S(t) I(t)+(1-\theta) \beta I(t) \\
& \rho(0, \tau)=\phi(\tau) \\
& S(0)=S_{0} ; I(0)=I_{0}
\end{aligned}
$$

where

$\beta \quad$ natural birth rate for the population; $\mu$ natural death rate for the population $\alpha \quad$ rate of contracting the HIV virus; $\sigma(\tau)$ death rate due to HIV virus infection $\delta \quad$ constant; $k$ is the measure of the efficacy of the anti-retroviral therapy (ART); $\theta$ is the proportion of the off-springs of the infected who are virus-free at birth; $0 \leq \theta \leq 1 . t$ is time; $\tau$ is the infection age and $T$ is the maximum infection age, We re-write (1)-(7) as follows

$$
\begin{aligned}
& \frac{d S(t)}{d t}=(\beta-\mu) S(t)+\theta \beta I(t)-\alpha S(t) I(t) \\
& \frac{\partial i(t, \tau)}{\partial t}+\frac{\partial i(t, \tau)}{\partial \tau}+(\mu+\sigma(\tau)) i(t, \tau)=0 \\
& \sigma(\tau)=\delta_{1} e^{-k(T-\tau)} \\
& I(t)=\int_{0}^{T} i(t, \tau) d \tau \\
& i(t, 0)=B(t)=\alpha S(t) I(t)+(1-\theta) \beta I(t) \\
& i(0, \tau)=i(\tau) \\
& S(0)=S_{0} ; I(0)=I_{0}
\end{aligned}
$$

\section{RESULTS AND DISCUSSION}

We present the results of our stability analysis of the endemic equilibrium state namely: the local and global stability of the endemic equilibrium state.

Equilibrium State: At the endemic equilibrium state, we set

$$
\begin{aligned}
& S(t)=x^{*}, \quad I(t)=z^{*} \\
& i(t, \tau)=i^{*}(\tau)
\end{aligned}
$$

and so

$$
\begin{aligned}
& z^{*}=\int_{0}^{T} i^{*}(\tau) d \tau \\
& i^{*}(0)=B(0)=\alpha x^{*} z^{*}+(1-\theta) \beta z^{*}
\end{aligned}
$$

substituting (15)-(17) into (8), (9) and (12) give

$$
\begin{aligned}
& (\beta-\mu) x^{*}+\theta \beta z^{*}-\alpha x^{*} z^{*}=0 \\
& \frac{d i^{*}(\tau)}{d \tau}+h(\tau) i^{*}(\tau)=0
\end{aligned}
$$

where

$h(\tau)=(\mu+\sigma(\tau))$

$i^{*}(0)=\alpha x^{*} z^{*}+(1-\theta) \beta z^{*}$

From (19) we obtain

$z^{*}=\frac{(\beta-\mu) x^{*}}{\alpha x^{*}-\theta \beta}$

From (20) we obtain

$i^{*}(\tau)=i^{*}(0) \exp \left\{-\int_{0}^{\tau} h(s) d s\right\}$

Integrating (24) over the interval $[0, T]$

and using (18) give

$z^{*}=\left[\alpha x^{*} z^{*}+(1-\theta) \beta z^{*}\right] A$

where

$A=\int_{0}^{T}\left\{\exp \left[-\int_{0}^{\tau} h(s) d s\right]\right\}$

From (25) we get

$x^{*}=\frac{1-(1-\theta) \beta A}{\alpha A}$

substituting (27) into (23) give

$z^{*}=\frac{(\mu-\beta)[1-(1-\theta) \beta A]}{\alpha(\beta A-1)}$

From (20) we get

$i^{*}(\tau)=i^{*}(0) \pi(\tau)$

where

$\pi(\tau)=e^{-\mu \tau} e^{-\int_{0}^{\tau} \sigma(s) d s}$

Basic Reproduction Number: The basic reproduction number is one of the fundamental concepts in mathematical biology that determines the future of an epidemic. According to Diekmann et al. (2000) and Murray (2002), the basic reproduction number denoted by $R_{0}$ is the expected number of secondary cases produced, in a completely susceptible population, by a typical infective individual. It is one of the most useful threshold parameters, which characterize mathematical problems concerning infectious diseases. 
If $R_{0}<1$, this implies that, on average an infected individual produces less than one new infected individual during the infectious period and the infection can be wiped out. Conversely, if $R_{0}>1$, then, each infected individual produces, on average, more than one new infection, and the disease is spread in the population. For a single infected compartment, $R_{0}$ is simple the product of the

$$
R_{0}=\alpha x \int_{0}^{T} \pi(\tau) d \tau+(1-\theta) \beta \int_{0}^{T} \pi(\tau) d \tau
$$

\section{Local Stability of the Endemic Equilibrium State.}

Lemma 1: The unique endemic equilibrium $E^{*}\left(x^{*}, i^{*}(\tau)\right)$ given by (27) and (29) with $i^{*}(0)$ given by (22) is locally asymptotically stable if $R_{0}>1$.

Proof: To show the local stability we linearize the system (8)-(12) around the endemic equilibrium $E^{*}$.In particular we set

$$
\begin{aligned}
& S(t)=x^{*}+x(t) \\
& i(t, \tau)=i^{*}(\tau)+z(t, \tau)
\end{aligned}
$$

We look for solutions of the linearized system in exponential form $x(t)=x e^{\lambda t}$ and $z(t, \tau)=z(\tau) e^{\lambda t}$

From (8) we have that

$$
\begin{aligned}
& \frac{d S(t)}{d t}=\beta S(t)-\mu S(t)+\theta \beta I(t)-\alpha S(t) I(t) \\
& \frac{d x(t)}{d t}=\beta\left(x^{*}+x(t)\right)-\mu\left(x^{*}+x(t)\right) \\
& +\theta \beta \int_{0}^{T}\left[i^{*}(\tau)+z(t, \tau)\right] d \tau \\
& -\alpha\left(x^{*}+x(t)\right) \int_{0}^{T}\left[i^{*}(\tau)+z(t, \tau)\right] d \tau
\end{aligned}
$$

infection rate and the mean duration of the infection. But for complicated models, this simple definition of $R_{0}$ is insufficient. For infection-age-structured models, the basic reproduction number is defined as the sum of the total infectivity of each infected compartment. In other words the total infectivity's give the basic reproduction number. In view of this, our basic reproduction number becomes:

$$
\frac{\partial z(t, \tau)}{\partial t}+\frac{\partial z(t, \tau)}{\partial \tau}+(\mu+\sigma(\tau)) z(t, \tau)=0
$$

or

$$
\frac{d z(\tau)}{d \tau}+(\lambda+\mu+\sigma(\tau)) z(\tau)=0
$$

From (38) we obtain;

$$
z(\tau)=z(0) e^{-\lambda t} \pi(\tau)
$$

where

$\pi(\tau)=e^{-\mu \tau} e^{-\int_{0}^{\tau} \sigma(s) d s}$

using (18), (32) and (33) in (12) and simplifying gives

$$
\begin{aligned}
& z(0)=i^{*}(0)+\alpha x z^{*}+\alpha x x_{0}^{*} z(\tau) d \tau \\
& +\alpha x \int_{0}^{T} z(\tau) d \tau+(1-\theta) \beta \int_{0}^{T} z(\tau) d \tau
\end{aligned}
$$

adding (36) and (41) give,

$$
\begin{gathered}
(\lambda+\mu-\beta) x=i^{*}(0)-z(0)+\beta \int_{0}^{T} z(\tau) d \tau \\
\text { or } x=\frac{i^{*}(0)-z(0)+\beta \int_{0}^{T} z(\tau) d \tau}{(\lambda+\mu-\beta)}
\end{gathered}
$$

substituting (42) into (41) and using (39) give

$$
\begin{aligned}
& z(0)=i^{*}(0)+\alpha x \int_{0}^{*} z(0) e^{-\lambda \tau} \pi(\tau) d \tau \\
& +(1-\theta) \beta \int_{0}^{T} z(0) e^{-\lambda \tau} \pi(\tau) d \tau
\end{aligned}
$$




$$
\begin{aligned}
& +\alpha z^{*}\left(\frac{i^{*}(0)-z(0)+\beta \int_{0}^{T} z(0) e^{-\lambda \tau} \pi(\tau) d \tau}{(\lambda+\mu-\beta)}\right) \\
& +\alpha\left(\frac{i^{*}(0)-z(0)+\beta \int_{0}^{T} z(0) e^{-\lambda \tau} \pi(\tau) d \tau}{(\lambda+\mu-\beta)}\right) \int_{0}^{T} z(0) e^{-\lambda \tau} \pi(\tau) d \tau
\end{aligned}
$$

or

$$
\begin{aligned}
& 1=\frac{i^{*}(0)}{z(0)}+\alpha x^{*} \int_{0}^{T} e^{-\lambda \tau} \pi(\tau) d \tau \\
& +(1-\theta) \beta \int_{0}^{T} e^{-\lambda \tau} \pi(\tau) d \tau \\
& +\alpha z^{*}\left(\frac{i^{*}(0)-z(0)+\beta \int_{0}^{T} e^{-\lambda \tau} \pi(\tau) d \tau}{(\lambda+\mu-\beta)}\right) \\
& +\alpha\left(\frac{i^{*}(0)-z(0)+\beta \int_{0}^{T} z(0) e^{-\lambda \tau} \pi(\tau) d \tau}{(\lambda+\mu-\beta)}\right) \int_{0}^{T} e^{-\lambda \tau} \pi(\tau) d \tau
\end{aligned}
$$

We note that when $\lambda$ is real and $i^{*}(0)<z(0)$, then the right hand side of (43) is a decreasing function of $\lambda$. Therefore, equation (43) has a solution $\lambda>0$ if $R_{0}>1$.

The local asymptotic stability of the endemic equilibrium $E^{*}$ is completed by observing that the real root of (43) has the dominant real part and this is obtained by considering absolute values as in Ashezua et al. (2015). This completes the proof of the lemma.

\section{Global Stability of the Endemic Equilibrium State:}

Before we prove the global stability of the endemic equilibrium state, we state the following lemma as outlined in the works of Brauer et al. (2013).
Let $V_{3}=\int_{0}^{T} \Psi(a) G\left(p(t, a), p^{*}(a)\right) d \tau$. Then

$$
V_{3}^{\prime}=\Psi(0) G\left(p(t, 0), p^{*}(a)\right.
$$

$+\int_{0}^{T}\left(\Psi^{\prime}(a)-\delta(a) \Psi(a)\right) G\left(p(t, a), p^{*}(a)\right) d a$

We construct the following Lyapunov functional following the approach as outlined in Huang et al. (2012) and Brauer et al. (2013).

$$
V=V_{1}+V_{2}
$$

where

$$
V_{1}=\left(x-x^{*}-x^{*} \ln \frac{x}{x^{*}}\right)
$$

and

$$
V_{2}=\int_{0}^{T} i^{*}(\tau) G\left(i(t, \tau), i^{*}(\tau)\right) d \tau
$$

Direct differentiation of (45) and using (8) gives,

$$
\frac{d V_{1}}{d t}=-\left(\mu+\alpha z^{*}\right)\left(x-x^{*}\right)+\left(\beta+\frac{\theta \beta z^{*}}{x}\right)\left(x-x^{*}\right)
$$

Direct differentiation of (46) using lemma 2 gives

$$
\begin{aligned}
& \frac{d V_{2}}{d t}=i^{*}(0) G\left(i(t, 0), i^{*}(0)\right) \\
& +\int_{0}^{T}\left(\frac{d i^{*}(\tau)}{d t}-h(\tau) i^{*}(\tau)\right) G\left(i(t, \tau), i^{*}(\tau)\right) d \tau
\end{aligned}
$$

Using (20) in (48) gives

$$
\begin{aligned}
& \frac{d V_{2}}{d t}=i^{*}(0)\left(1-\frac{i(t, 0)}{i^{*}(0)}+\ln \frac{i(t, 0)}{i^{*}(0)}\right) \\
& +2 \int_{0}^{T} \frac{d i^{*}(\tau)}{d t}\left(1-\frac{i(t, 0)}{i^{*}(\tau)}+\ln \frac{i(t, 0)}{i^{*}(\tau)}\right) d \tau \\
& \text { adding (47) and (49) gives } \\
& \frac{d V(t)}{d t}=X-Y+Z
\end{aligned}
$$

where

$$
\begin{aligned}
& X=\left(\beta+\frac{\theta \beta z^{*}}{x}\right)\left(x-x^{*}\right) \\
& Y=(\mu+\alpha I)\left(x-x^{*}\right)
\end{aligned}
$$

\section{Lemma2:}




$$
\begin{aligned}
& Z=i^{*}(0)\left(1-\frac{i(t, 0)}{i^{*}(0)}-\ln \frac{i(t, 0)}{i^{*}(0)}\right) \\
& +2 \int_{0}^{T} \frac{d i^{*}(\tau)}{d t}\left(1-\frac{i(t, \tau)}{i^{*}(\tau)}-\ln \frac{i(t, \tau)}{i^{*}(\tau)}\right) d \tau
\end{aligned}
$$

From (53) we see that $Z \leq 0$ since $1-x+\ln x \leq 0$ for all $x>0$ with equality holding if and only if $x=1$. From (50), if $X<Y$ then $\frac{d V(t)}{d t}$ will be negative definite, meaning that $\frac{d V(t)}{d t}<0$. Also it

Acknowledgement: The first author wishes to thank the Federal University of Agriculture, Makurdi, Nigeria for granting him study fellowship during which this research work was carried out.

\section{REFERENCES}

Akinwande, N. I. (2005). A mathematical model of the chaotic dynamics of the AIDS disease pandemic. J. Nig. Math. Soc., 24, 8-16.

Akinwande, N. I. (1999). On the characteristics equation of a non-linear age-structured population model; ICTP, Trieste, Italy Preprint IC/99/153.

Diekmann, O., Heesterbeek, J. A. P. and Metz, J. A. J. (2000): Mathematical epidemiology of infectious diseases. Model Building, Analysis and Interpretation Wiley Series in Mathematical and Computational Biology, John Wiley and Sons, La., Chichester. follows that, $\frac{d V(t)}{d t}=0$ if and only if $x=x^{*}$ and $\frac{i(t, 0)}{i^{*}(0)}=\frac{i(t, \tau)}{i^{*}(\tau)}$, for all $\tau \geq 0$. Therefore the largest compact invariant set in $\left\{\left(x^{*}, i^{*}(\tau)\right) \in \Omega: \frac{d V(t)}{d t}=0\right\} \quad$ is the singleton $\left\{E^{*}\right\}$. If $R_{0}>1$, then by the LaSalles's invariant principle, $E^{*}$ is globally asymptotically stable in $\Omega$ if $X<Y$.

Ashezua, T. T., Akinwande, N. I., Abdulrahman, S., Olayiwola, R. O. \& Kuta, F. A. (2015). Local stability analysis of an infection age mathematical model for tuberculosis disease dynamics. J. Appl. Sci. Environ. Manage., 19(4), 665-669.

Bellman, R. and Cook, K. L. (1963). Differential difference equations, Academic Press, London.

Brauer, F., Shuai, Z. and Van Den Driessche (2013). Dynamics of an age-of-infection cholera model. Mathematical Biosciences and Engineering, 10(5 \&6), 1335-1349.

Huang, G., Liu, X. and Takeuchi, Y. (2012). Lyapunov functions and global stability for age-structured HIV infection model, SIAM J. Appl. Math., 72, 25-38.

Murray, J. D. (2002): Mathematical Biology, an introduction, 17, Springer, New York, third edition. 